\title{
Regionalpolitischer Beitrag der Technologiepolitik
}

\section{Problemstellung}

Wohlstand und Erfolg von Wirtschaft und Gesellschaft am Standort Schweiz hängen immer mehr von Technologien und ihrem Management ab. Für viele Unternehmen ist Wettbewerb über Qualität und Neuerungen ein noch wichtigeres Element ihrer Strategie als der Preis-/ Kostenwettbewerb. Ihr Erfolg hängt ganz wesentlich von der erfolgreichen Anwendung und Umsetzung von Technologie, oft auch kombiniert mit Diensten, am Markt ab. Ihre Anwendungskompetenz muß gestärkt werden.

Technologiepolitik soll die Entstehung sowie die rasche Umsetzung und Verbreitung von neuem, technikrelevantem Wissen und Können zwecks wirtschaftlichem Erfolg am Markt unterstützen. Damit liegt der Primat auf der Wirtschaftspolitik; Technologiepolitik wird als technologieorientierte Wirtschaftspolitik verstanden, in Abgrenzung zur Forschungs-, Wissenschafts- und Bildungspolitik.

Im Zentrum der regionalpolitischen Diskussion standen lange Zeit regionale Disparitäten und der (nachträgliche) Ausgleich der Entwicklung in den einzelnen Regionen, das Verteilungsziel. Doch: die traditionellen Regionen sind nicht mehr so homogen wie bisher. "Die räumlichen Disparitäten entsprechen in der Schweiz nicht mehr dem dualen Bild (Berggebiet - Talgebiet) oder (Zentrum - Peripherie) der frühen siebziger Jahre. Die feststellbaren Unterschiede auf kleinräumiger Ebene sind mosaikartig geworden», hält die Beratende Kommission für regionale Wirtschaftsförderung fest und fährt weiter: «Neben den für die interkommunale Zusammenarbeit zweckmäßigen kleinräumigen Regionen rücken in einer sich öffnenden Schweiz größere, auch überkantonale Gebietseinheiten vermehrt in den Blickpunkt.»

Aufgrund dieser Befunde drängt sich eine neue Perspektive der Regionalpolitik auf: weg vom dualen Bild der Schweiz hin zu mehreren Großregionen aufgrund von wirtschaftlich-funktionalen Zusammenhängen. Im Sinne eines Perspektivenwandels geht es neu um Aspekte der (internationalen) Wettbewerbsfähigkeit und der Stand ortattraktivität und damit um das Ziel der Allokationseffizienz verbunden mit der Umweltverträglichkeit. Allerdings darf das Ausgleichsziel bei dieser Neuorientierung nicht völlig vernachlässigt werden.

Aufgrund dieser Überlegungen besteht ein sachlogischer Zusammenhang zwischen Technologie- und Regionalpolitik in der Verbindung von rascher Diffusion resp. Umsetzung von Technologien und optimaler Faktoral- lokation. Dabei sollen beide die Bewältigung künftiger Herausforderungen und den aktiven Strukturwandel unterstützen.

\section{Grundsätze der regionalpolitischen Ausgestaltung der Technologiepolitik}

Die Wettbewerbsfähigkeit und Innovationskraft von Branchen, Industrien und Volkswirtschaften hängen nicht nur von den Fähigkeiten einzelner Firmen und Individuen ab. Große und kleine Firmen, Forschungs- und Entwicklungsstätten, Zulieferer und Abnehmer und verschiedene Dienstleister stehen nicht nur in einem Wettbewerbsverhältnis im Sinne der neoklassischen Ökonomie miteinander. Bei Innovationsprozessen erfüllen sie auch einander ergänzende Funktionen und bilden zusammen mehr als die Summe der einzelnen Aktoren. Schulen bilden Leute für Unternehmen aus, sind über Forschungs- und Entwicklungsprojekte oder Beratungsleistungen mit ihnen verbunden und empfangen von diesen ihrerseits konkrete Signale. Die Unternehmen sind untereinander als Zulieferer und Abnehmer, aber auch als kooperierende Partner auf gleicher Fertigungsstufe vernetzt. Viele wettbewerbsfähige Leistungen werden auf diese Weise in Zusammenarbeit erbracht. Die Innovationskraft von Industrien, Branchen und Volkswirtschaften beruht deshalb ganz entscheidend auf der Qualität des Zusammenwirkens verschiedener Akteure. Wichtig ist die Fähigkeit von Wirtschaft, Bildung/Wissenschaft und Staat, Teams oder problemorientierte Kooperationspartnerschaften zu bilden. Das Resultat wird vielfach als cluster oder als milieu innovateur bezeichnet. Wir sprechen von Leistungsverbundsystemen.

In den an Technologien orientierten Verbundsystemen der Schweiz sollten folgende Institutionen vernetzt sein:

- Industrie- und Dienstleistungsunternehmen (insbesondere KMU) sowie Industrielabors,

- zu Fachhochschulen auszubauende Ingenieurschulen/ HTL und HWV sowie regionale Technikerschulen, Gewerbe- und Berufsschulen und die zuständigen Behörden,

- die beiden ETH und ihre Annexanstalten sowie kantonale Universitäten,

Beat Hotz-Hart, Prof. Dr., Vizedirektor Bundesamt für Konjunkturfragen, Belpstraße 53, 3003 Bern 
- Kompetenzzentren, wie sie im Bereich CIM oder Mikroelektronik im Rahmen von Aktionsprogrammen durch das Bundesamt für Konjunkturfragen (BfK) aufgebaut worden sind, ihre Leitungsorgane und Trägerorganisationen,

- bereits bestehende Innovationsberatungs- und Technologietransferstellen,

- entsprechende Aktivitäten der regionalen und kantonalen Wirtschaftsförderung,

- Technoparks.

Technologische Impulsgeber und Moderatoren dieser Verbundsysteme sind - im günstigsten Falle - die in den Regionen verankerten Kompetenzzentren und HTL/Fachhochschulen. Diese sollen eine Multiplikatorwirkung auf die Region auslösen, Prozesse über Firmengrenzen hinweg anstoßen und unternehmerisch stärken und eine gesunde Durchmischung und Regionalisierung der Wirtschaft fördern. Dafür und zum Zwecke der Steigerung der technologischen Kompetenz und der Innovationskraft in der Region haben sie einen dreifachen Leistungsauftrag. Aus- und Weiterbildung, anwendungsorientierte Entwicklungsarbeiten sowie Dienstleistungen für Dritte, insbesondere Technologie- und Wissenstransfer.

Weiter tragen auch Austauschbeziehungen zwischen diesen großregionalen Verbundsystemen zur erfolgreichen Wirtschaftsentwicklung bei. Deshalb sind auch interregionale, nationale, ja europäische Verbindungen zwischen den aufgeführten Institutionen zu beachten. Daraus können sich nationale Schwerpunkte bilden.

Die technologieorientierten großregionalen Verbundsysteme sind nicht fix abgegrenzt. Vielmehr sind sie im Sinne einer "géometrie variable» den spezifischen $\mathrm{Be}$ dürfnissen der einzelnen Aufgaben angepaßt. Sie können sich auch überlappen (vgl. z. B. die bestehenden Netzwerke in den Technologiebereichen Mikrosystemtechnik, Tribologie oder CIM). Auch sind ihre Netzwerke flexibel und auf Zeit angelegt.

Nach dem Netzwerkgedanken sind technologiepolitische Instrumente zu entwickeln, die nicht ausschließlich und isoliert bei einzelnen Aktoren ansetzen. Vielmehr sollten sie zu ihrer Vernetzung, zur Herstellung eines problem- resp. marktorientierten Zusammenhanges in einem System beitragen. Sie sollen sich auf Prozesse konzentrieren wie Kooperationen stiften und helfen, Synergien zu nutzen. Es geht darum, gerade auch räumlich geeignete Konstellationen von technischer Kompetenz und industriellem Pioniergeist herzustellen oder zu stärken und damit regionale Potentiale zu mobilisieren und zur Dynamik und Wettbewerbsfähigkeit der Region beizutragen.

\section{Politikmaßnahmen}

Der Netzwerkansatz läuft damit im wesentlichen auf die Schaffung, Stärkung und Dynamisierung innovativer Milieus ("clusters») oder technologieorientierter Verbundsysteme aus Unternehmen, Aus- und Weiterbildungs- sowie Technikentwicklungsstätten und staatli- chen Stellen im regionalen, nationalen und internationalen Verbund hinaus. Dies ist wesentlich eine private Aufgabe, soll jedoch durch die Politik unterstützt und moderiert werden.

Die Intensität des Engagements des Bundes im Sinne der Beeinflussung der Bildung und Entwicklung solcher Verbundsysteme kann unterschiedlich sein. Sie kann variieren zwischen Basisfinanzierung, verbunden mit Nichteinmischung und Hoffen auf Selbstkoordination zwischen und innerhalb der Regionen, Mitwirkung, Beeinflussung wichtiger Entscheide wie Standortwahl, Regionenbildung, inhaltliche Schwerpunktsetzung und organisatorischer Leitung. Die Wahl der angestrebten Intensität ist eine politisch zu entscheidende Frage. Dabei sollte immer das Ziel der Steigerung der Wettbewerbsfähigkeit, also der Beitrag zur Allokationseffizienz und Technikumsetzung und -diffusion, im Auge behalten werden.

Folgende instrumentelle Dimensionen bieten sich bundesseitig für die Umsetzung des skizzierten Politikkonzeptes an. Dabei kann vor allem an die Erfahrungen mit den Aktionsprogrammen des Bundesamtes für Konjunkturfragen angeknüpft werden.

- Moderation und Organisation des regionalen und nationalen Prozesses: Den Bundesinstanzen ist eine aktive Moderatorenrolle im technologie- und raumordnungspolitischen Prozeß (Lancierung von Ideen, Veranstaltung von Wettbewerb zwischen den Regionen, Zusammenbringen verschiedener Stufen und Gruppierungen, Vermittlung bei divergierenden Interessen usw.) zu übertragen. Sie veranstalten in technologierelevanten Bereichen Innovationswettbewerb, organisieren und vernetzen thematisch orientierte Debatten, betreiben Projektförderung über Ausschreibungen, stiften Kooperationen, setzen über Wettbewerb Leistungsstandards und versuchen auf verschiedenen Wegen, einen volkswirtschaftlichen Hebeleffekt zu erzielen. Dies ist keine bloße Subventionspolitik, sondern eine qualitativ hochstehende Dienstleistung. Damit wird mit relativ wenig Geld viel bewirkt. Der volkswirtschaftliche Grenznutzen solcher Anstrengungen ist im Vergleich zu vielen anderen Politikbereichen deutlich höher. Unter den Restriktionen der dramatischen Finanzknappheit bei der öffentlichen Hand und gemessen an ihrer hohen Effektivität, sollte gerade einer solchen Politik mehr Beachtung und Gewicht gegeben werden.

- Einflußnahme auf Standortentscheide: Auf die räumliche Verteilung von Fachhochschulen oder Kompetenzzentren, auf die thematische Schwerpunktbildung im Lehrangebot und der F\&E-Aktivitäten sowie der Dienstleistungen an solchen Institutionen und Standorten ist von Bundesseite Einfluß zu nehmen. Dazu sind Kriterien und Verfahren zu entwickeln und durchzusetzen.

- Gemischte Finanzierung durch Bund, Kantone und Privatwirtschaft: Die Aktionsprogramme CIM und MICROSWISS des Bundesamtes für Konjunkturfragen zeigen: Die Finanzierung von Projekten durch Beiträge von Bund und Kantonen und der Wirtschaft, die Mischfinan- 
zierung, hat sich bewährt. Wer von Leistungen des Bundes profitieren will, muß einen angemessenen Eigenbeitrag leisten. Der Bund vermittelt Anstöße, mobilisiert Kräfte in den Regionen und trägt zu ihrer nationalen Bündelung bei. Dabei orientiert er sich nicht an einem einheitlichen Bundessatz, sondern an Leistungskriterien, dieflexibel gehandhabt werden können. Bundesleistungen an Vorhaben im Rahmen der regionalpolitisch ausgerichteten Technologiepolitik sollten u. a. davon abhängig gemacht werden, ob diese großräumig koordiniert und abgestimmt sind und eine wirksame "Ausstrahlung» in die Region erzielen. Allerdings muß der Bund gerade in finanzpolitisch schwierigen Zeiten ein verläßlicher Partner sein und Kontinuität in finanzieller Hinsicht gewährleisten.

- Mix von Basisfinanzierung und Förderung von guten, anwendungsorientierten Projekten: Der Bund soll einen angemessenen Teil der Basisfinanzierung von Teilnehmern an einem Technologieverbund übernehmen. Ein substantieller Teil seiner Unterstützung soll jedoch über die Förderung von guten, anwendungsorientierten Projekten von Teilnehmern aus dem Verbund geschehen. Dabei müssen Kriterien der Förderinstanzen des Bundes konkret der Kommission für Technologie und Innovation (KTI, vormals KWF) - erfüllt werden.
Projektorientiertes Vorgehen und Projektmanagement sollen nicht nur für F \& E gelten, sondern auch im Ausund Weiterbildungsbereich: der Bund sollte vermehrt eine Leistungsorientierung für Institutionen durch stärkeren Einsatz von projektbezogener Finanzierung anstreben. Damit sollen Wettbewerb und ein unternehmerischer Geist in die Schulen und F \& E-Einrichtungen getragen werden.

Das Netzwerk von Unternehmen, Hochschulen, Fachhochschulen, Technologie-Kompetenzzentren sowie Innovationsberatungsstellen und Technologieparks ist vertikal und horizontal weiter zu knüpfen. Klare regionale Knoten sollen vom Bund durch projektweise Förderung von $\mathrm{F} \& \mathrm{E}$ gestärkt werden. Damit soll das vorhandene und schlummernde Potential in den Regionen mobilisiert und ein Klima der offensiven Kooperation geschaffen werden. Gelingt es, ein solches Netzwerk aufzubauen, mit Leben zu füllen und das regulatorische Umfeld wesentlich zu verbessern, so ist die Chance deutlich größer, daß sich der Industriestandort Schweiz im internationalen Wettbewerb behaupten kann. Die Technologiepolitik leistet damit einen substantiellen Beitrag zur Erhaltung und Schaffung von attraktiven Arbeitsplätzen und Einkommen am Standort Schweiz.

\section{Das Berggebiet und die Neuorientierung der schweizerischen Regionalpolitik}

Vier Eigenschaften charakterisieren die Neuorientierung der schweizerischen Regionalpolitik: Das Berggebiet bleibt primäres Zielgebiet der meisten regionalpolitischen Instrumente, die kleinräumige (IHG-Regionen) wird durch eine großräumige (Kantone, Grenzregionen) Regionalpolitik ergänzt, zu den kohäsionspolitischen Instrumenten (IHG, REGIO PLUS) kommen integrationspolitische (INTERREG) hinzu, und der nachhinkende Disparitätenabbau soll durch die Förderung der regionalen Anpassungs- und Wettbewerbsfähigkeit abgelöst werden.

Diese Zieloptik ist konsistent mit der marktwirtschaftlichen Erneuerung des Wirtschaftsstandortes Schweiz als Antwort auf die globalwirtschaftlichen Herausforderungen. Die rasche Vervielfachung qualifizierter und differenzierter Standorte für die Wirtschaft und die neuen wirtschaftspolitischen Vorgaben durch die GATT/WTORegeln sowie die fortschreitende Integration großer
Wirtschaftsräume (Triade: Europa, Nordamerika, Südostasien) erfordern innovationsfördernde Rahmenbedingungen und wettbewerbsfähige Standorte in der Schweiz.

Die Regionalpolitik soll in erster Linie die wirtschaftliche Erneuerungsfähigkeit unterstützen, indem sie für diese Aufgaben geeignete Trägerschaften und Projekte mitfinanziert sowie offene und vernetzte Regionsstrukturen fördert. In diesem Dispositiv nehmen die Kantone als Träger der klein- und großräumigen Regionalpolitik eine Schlüsselrolle ein. Diese Position soll insbesondere durch die Neuordnung des bundesstaatlichen Finanz-

Paul Messerli, Prof. Dr., Geographisches Institut, Universität Bern, Hallerstraße 12, 3012 Bern 